P80 INTERSECTIONAL SOCIAL IDENTITIES, DISCRIMINATION AND MENTAL HEALTH SERVICE: FINDINGS FROM THE 2014 ADULT PSYCHIATRIC MORBIDITY SURVEY OF ENGLAND

'S Hatch, ${ }^{1} \mathrm{C}$ Woodhead*, 'R Rhead, 'Z Chui, ${ }^{2}$ S McManus, '1 Das-Munshi, ' 'G Ahmad, ${ }^{1} \mathrm{H}$ Harwood. 'Psychological Medicine, King's College Hospital, London, UK; ${ }^{2}$ The National Centre for Social Research (NATCen), London, UK

\subsection{6/jech-2020-SSMabstracts. 172}

Background Internationally there is a large treatment gap for common mental disorders and disparities across population groups but little understanding about whether people occupying multiple advantaged/disadvantaged statuses access mental health support differentially. This study examines discrimination and mental health service use (MHSU) at the intersections of social statuses.

We hypothesised i) greater past-year discrimination among disadvantaged social statuses; ii) different patterns of MHSU/ treatment among single, compared to multiple social status groups; and after accounting for need: iii) less MHSU/treatment among multiply disadvantaged than more advantaged social status groups; and, iv) less MHSU/treatment among those reporting discrimination.

Methods English population-based data came from the 2014 Adult Psychiatric Morbidity Survey. Latent Class Analysis (LCA) was used to define intersectional social status groups. Multivariate logistic regression models were estimated to examine associations between MHSU/treatment and i) single social statuses and ii) latent classes of social status by gender, adjusting for confounders and need (physical/mental illness), followed by exposure to past year discrimination.

Results Five-class LCA solutions were selected for men and women, characterised as: 1) retired White British; 2) employed migrants, 3) economically inactive migrants 4) employed White British, lower education/social class; and 5) employed White British, high education/social class. Discrimination was more common among disadvantaged groups, and patterns differed across single and intersectional analyses. After adjustments, MHSU/treatment was elevated among females (OR 1.88, 95\% CI: 1.49-2.06) and sexual minorities (OR 1.63: 1.06-2.51) but lower among Black ethnic groups (OR 0.28: 0.14-0.56). Adjustments for discrimination attenuated associations for sexual minorities and Black respondents. Intersectionally, findings were similar by gender except the retired White British group, for whom MHSU/treatment was significantly elevated among women (OR 1.96: 1.32-2.90) but not men. Among women and men respectively, compared to the most advantaged group, greater odds of MHSU/treatment were found for 'employed migrants' (OR 2.50: 1.71-3.67; OR 2.56: 1.77-3.71), 'economically inactive migrants' (OR 4.47: 3.00-6.40; OR 4.60: 3.10-6.83) and 'employed White British, low social class/education' (OR 1.91: 1.32-2.79; OR 1.95: 1.43-2.63). Adjustments for discrimination had little influence.

Conclusion Accounting for need, MHSU/treatment disparities are apparent but differ when considering single, or multiple social statuses. Single status analyses mask discrepancies observed intersectionally, while intersectional data-driven analyses miss inequities by minority statuses which do not distinguish latent classes. For some groups, discrimination may elevate, rather than inhibit MHSU/treatment. To better inform policy and practice, research should incorporate multiple and mixed-methods approaches to identify complexities of social stratification processes.

\section{P81 SOCIAL PATTERNING OF SELF-REPORTED HEALTH AMONG 10-24-YEAR-OLDS ACROSS THE UK COUNTRIES: A PRELIMINARY ANALYSIS OF THE 2001 AND 2011 CENSUSES}

${ }^{1} \mathrm{~K}$ Metsis*, ${ }^{1,2} \mathrm{~J}$ Inchley, ${ }^{1} \mathrm{~F}$ Sullivan. 'School of Medicine, University of St Andrews, St Andrews, UK; ${ }^{2}$ MRC/CSO Social and Public Health Sciences Unit, University of Glasgow, Glasgow, UK

\subsection{6/jech-2020-SSMabstracts. 173}

Background Scotland has higher rates of mortality and morbidity compared to other UK countries. These differences are partially explained by socio-economic factors. It is acknowledged that adult health is strongly influenced during childhood and adolescence, however, because adolescence is considered to be the healthiest period in one's life, research into health inequalities has largely focused on younger children and the adult population. This study investigates the social patterning of self-reported health among 10-24-yearolds across the four UK nations.

Methods We used 2001 and 2011 census microdata from the four UK's constituent countries to examine social patterning of self-reported health by UK country, gender, social position (operationalised by the NS-SEC of the family reference person), and household deprivation indicators of education and housing. Health was measured by using the general health question 'over the last twelve months would you say your health has on the whole been: good, fairly good, not good'? Preliminary results from logistic regression analysis are presented here. The final results will be reported in the form of marginal effects.

Results Analysis of the 2001 data so far shows significant differences in self-reported health by the country, gender, NS-SEC, and household education. After controlling for the effect of social position, gender, education and housing, young people from Scotland and Northern Ireland have respectively $22 \%(95 \%$ CIs $0.72-0.85)(\mathrm{p}<0.001)$ and $17 \%$ (95\% CIs $0.73-0.96)(p=0.008)$ higher odds of reporting poor health compared to England. The social patterning of self-reported health is visible; respondents from semi-routine and routine occupations have 23\% higher odds $(95 \%$ CIs $0.65-0.92)(p=0.003)$ of reporting poor health compared to the NS-SEC Class 1 . Odds are also higher among respondents from 'residual' NS-SEC classes of 'never worked' (63\%, 95\% CIs $0.31-0.45)$ and 'long-term unemployed' (46\%, 95\% CIs $0.43-0.70)(\mathrm{p}<0.001)$. Young people from households with low educational attainment have 44\% higher odds of reporting poor health $(95 \%$ CIs $0.52-0.59$, $\mathrm{p}<0.001)$. Association between housing deprivation and health is not statistically significant. Analysis of the 2011 data is ongoing and full results will be available by the time of the conference.

Conclusion The results of the preliminary analysis confirm that the family's socio-economic status influences young people's self-reported health, the significant country effect will be further investigated. This study will also make a methodological contribution by comparing the results as reported in the form of odds ratios and marginal effects. 